Revue d'histoire de l'Amérique française

DEVIUE D.HISTOIRE DE L'AMÉRIQUE FRANÇAISE

\title{
Le contentieux de la Cour supérieure, 1880-1890 : droit, marché et société durant la transition au capitalisme industriel
}

\section{Thierry Nootens}

Volume 69, numéro 1-2, été-automne 2015

URI : https://id.erudit.org/iderudit/1034593ar

DOI : https://doi.org/10.7202/1034593ar

Aller au sommaire du numéro

\section{Éditeur(s)}

Institut d'histoire de l'Amérique française

\section{ISSN}

0035-2357 (imprimé)

1492-1383 (numérique)

Découvrir la revue

\section{Citer cet article}

Nootens, T. (2015). Le contentieux de la Cour supérieure, 1880-1890 : droit, marché et société durant la transition au capitalisme industriel. Revue d'histoire de l'Amérique française, 69(1-2), 165-188.

https://doi.org/10.7202/1034593ar
Résumé de l'article

Dans un article précédent, nous avons analysé la condition socioprofessionnelle des demandeurs et défendeurs impliqués dans les litiges entendus par la Cour supérieure dans le district de Trois-Rivières, durant la décennie 1880. Il s'agit maintenant d'examiner plus précisément l'éventail de ces affaires. Les conflits mettant en scène deux groupes spécifiques, soit les cultivateurs et les industriels, sont aussi mis en relief. Ce faisant, nous serons en mesure d'établir si le monde de la terre et l'entrepreneuriat capitaliste présentent un rapport au droit très spécifique à l'époque considérée. La question des interactions entre droit positif et expérience ordinaire du droit est abordée en conclusion générale. 


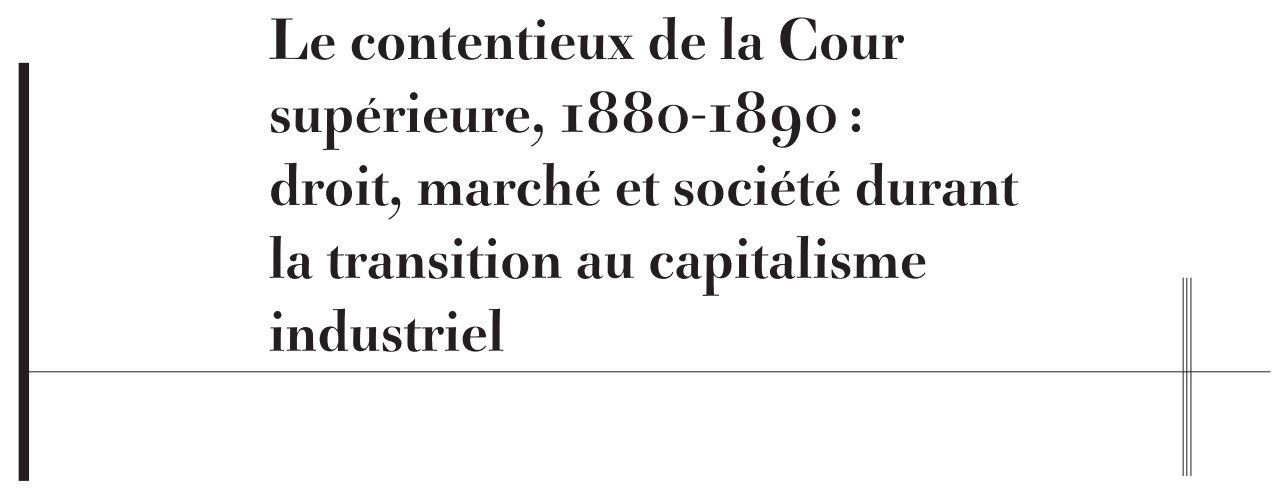

ThIERry NoOTENS

Département des sciences humaines

Université du Québec à Trois-Rivières

Centre interuniversitaire d'études québécoises (CIEQ)

RÉSUMÉ • Dans un article précédent, nous avons analysé la condition socioprofessionnelle des demandeurs et défendeurs impliqués dans les litiges entendus par la Cour supérieure dans le district de Trois-Rivières, durant la décennie 1880¹. II s'agit maintenant d'examiner plus précisément l'éventail de ces affaires. Les conflits mettant en scène deux groupes spécifiques, soit les cultivateurs et les industriels, sont aussi mis en relief. Ce faisant, nous serons en mesure d'établir si le monde de la terre et l'entrepreneuriat capitaliste présentent un rapport au droit très spécifique à l'époque considérée. La question des interactions entre droit positif et expérience ordinaire du droit est abordée en conclusion générale.

ABstract - We have examined in a previous paper the social status of plaintiffs and defendants involved in the cases heard by the Superior Court in the district of ThreeRivers during the 1880s. We now turn to the litigation in itself. Conflicts involving farmers and manufacturers are also highlighted. Thus, we will be able to establish if the countryside and the industrial sector show a specific relationship to law during this time. The issue of interactions between positive law and the ordinary experience of law is addressed in conclusion.

1. Thierry Nootens, «Les plaideurs en Cour supérieure, 1880-1890: classe, genre et juridicité durant la transition au capitalisme industriel ", RHAF, 68, 1-2 (2014): 25-56. 


\section{INTRODUCTION}

L'étude systématique du contentieux des tribunaux civils est rare en histoire du droit ${ }^{2}$. Les travaux de ce genre sont pour ainsi dire inexistants pour la période suivant le milieu du XIX ${ }^{\mathrm{e}}$ siècle. Dans un article précédent, nous avons présenté les premiers résultats d'une enquête menée parmi les 689 causes entendues par la Cour supérieure dans le district de TroisRivières durant les années 1880, 1885 et 1890. Les caractéristiques des 1853 parties demanderesses et défenderesses ont alors retenu notre attention. La surreprésentation des commerçants parmi les plaideurs et les effets judiciaires de l'infériorité juridique des femmes mariées ont été mis en lumière. L'analyse de la présence récurrente des mêmes individus dans le contentieux du tribunal a quant elle permis de mettre en évidence certaines réalités: le caractère interpersonnel des transactions conclues par les justiciables; le très fréquent report à échéance, grâce au crédit, du paiement effectif des mêmes transactions; l'influence considérable du patrimoine sur les rapports intrafamiliaux.

Pour bien comprendre, de concert, le rôle du droit dans la vie de ces justiciables et la tâche de régulation sociale qui échoit à la Cour supérieure, une présentation plus systématique de la panoplie de poursuites entamées devant cette instance s'impose. Quelles transactions et conflits conduisent les habitants du district judiciaire de Trois-Rivières devant ce tribunal? Que peut nous apprendre la lecture de ces affaires quant à la juridicisation des rapports sociaux durant la transition au capitalisme industriel, c'est-à-dire quant à la forme et à l'intensité des liens de droit qui structurent à cette époque les familles, communautés et réseaux d'affaires? Ce contentieux témoigne-t-il aussi d'une forme spécifique de judiciarisation? Il s'agit, sur ce plan, de caractériser le rôle du tribunal en matière d'arbitrage des disputes de tous ordres soumises à son attention.

Si le processus de réforme qui a abouti au Code civil du Bas-Canada de 1866 a été examiné par bien des chercheurs, peu d'entre eux ont tenté de rendre compte des fonctions assumées par le système judiciaire civil à partir du milieu du XIX ${ }^{\mathrm{e}}$ siècle. Rappelons que la Cour supérieure est créée en 1849 et que le nombre de districts judiciaires bondit en 1857 : ils passent de 7 à 19. On peut convenir, de manière générale, que ce resserrement du quadrillage judiciaire du territoire facilite l'accès aux tribunaux et, surtout,

2. Pour le détail des réalisations scientifiques en ce domaine, voir l'introduction de notre article précédent. 
qu'il intensifie la présence de l'État à l'échelle locale 3 . Ajoutons que la justice civile constitue un rouage important du développement du marché au XIX ${ }^{\mathrm{e}}$ siècle, puisque la multiplication et la complexification des transactions nécessitent un instrument de régulation sociale efficace, afin de départager les prétentions de tout un chacun et de sanctionner les manquements aux obligations contractées.

Ce point de vue n'est pas partagé par tous. L'étude des plaintes et tractations politiques relatives à l'incompétence et à l'absentéisme de certains juges a conduit Jonathan Swainger à soutenir que durant les années 1860 et 1870 , au lieu de créer une structure judiciaire rationnelle et propre à favoriser le capitalisme, la loi de 1857 «... institutionalized a fragmented and often chaotic system devoting considerable energy to managing an uneven collection of judicial talent ${ }^{4} »$. Si certains magistrats avaient des problèmes comportementaux, il faudrait valider cette thèse d'un «système chaotique » à la lumière d'un examen du travail concrètement accompli par les tribunaux. En outre, les juges, ou plus précisément quelques-uns d'entre eux, ne sont pas exactement le système judiciaire. Au surplus, comme on le verra ici, la Cour supérieure joue manifestement un rôle crucial dans le fonctionnement du marché durant la décennie 1880 .

Si les tribunaux facilitent le fonctionnement du marché, il convient de ne pas s'en tenir à une assertion aussi générale. Certes, un système judiciaire accessible - sur le plan des distances à parcourir, à tout le moins - est de nature à réduire les risques des opérations commerciales et, ce faisant, leur coût. Un tel système maximise donc l'efficacité économique, comme l'expliquent Peter George et Philip Sworden ${ }^{5}$. Néanmoins, il importe d'introduire dans l'équation «justice et économie» tout le dégradé des conditions sociales, à moins de souscrire à la représentation d'un marché fonctionnant de manière idéelle, comme le souhaitaient les élites politiques de l'époque. Un cultivateur aux abois et forcé de contracter un emprunt à taux élevé auprès d'un notable de sa paroisse ne transige pas exactement sur un marché libre d'entraves, de son point de vue.

L'éventail de litiges soumis à l'attention de la Cour supérieure dans le district de Trois-Rivières durant la décennie 1880 contient de bons indices en ce qui a trait aux rapports entre droit, justice, société et marché durant

\footnotetext{
3. Brian Young, The Politics of Codification: The Lower Canadian Civil Code of 1866 (Montréal et Kingston, McGill-Queen's University Press, 1994), 100-101.

4. Jonathan Swainger, "A Bench in Disarray: The Quebec Judiciary and The Federal Department of Justice, 1867-1878», Les Cahiers de Droit, 34, 1 (1993): 89.

5. Peter George et Philip Sworden, "The Courts and the Development of Trade in Upper Canada, 1830-1860", The Business History Review, 60, 2 (1986): 258-280.
} 
la seconde moitié du XIX siècle. Nous ferons état, pour débuter, de la manière dont une typologie des litiges a été mise sur pied. Les principales catégories d'affaires seront ensuite analysées. Pour finir, nous rendrons compte de la situation en justice de deux catégories sociales spécifiques, soit les cultivateurs et les industriels. Il sera possible, de ce fait, de comparer la juridicité et la litigiosité de deux pôles de la socio-économie du district: le monde rural et le secteur «moderne» de fructification du travail et de l'argent.

\section{UNE TYPOLOGIE DES AFFAIRES ENTENDUES PAR LA COUR}

La juridiction de la Cour supérieure couvre un large éventail de réalités sociales et institutionnelles, des transactions commerciales aux rapports familiaux, en passant par le travail accompli par les tribunaux inférieurs, pour ne nommer que quelques exemples. Tenir compte uniquement de la valeur en jeu aurait peu de sens. Les $100 \$$ réclamés comme pension alimentaire par un parent âgé et pauvre n'ont rien à voir, ni sociologiquement ni juridiquement, avec un compte en souffrance du même ordre dont un marchand de village réclame le paiement.

Cela dit, bon nombre de procès concernent des choses ou différents types de prestations qui se marchandent: biens procurés chez un commerçant, services offerts par des professionnels, immeubles. En d'autres temps, c'est un type précis d'engagement qui suscite une poursuite. C'est le cas des contrats et des billets promissoires. La régulation de la situation et du statut des individus constitue quant à elle un phénomène à part: les déconfitures financières et les affaires familiales - tant financières qu'interpersonnelles, ces deux réalités étant intimement liées - ont de ce fait été érigées en catégories spécifiques. Nous avons fait de même en ce qui concerne le pouvoir de surveillance de certains rouages de l'État comme les commissions scolaires et les municipalités. Les dommages en tous genres, enfin, représentent une forme particulière de litigiosité, en tant qu'accidents dans l'ordre des choses. Neuf catégories de litiges ont donc été établies. Une dixième et dernière catégorie rassemble les affaires dont le classement est plus malaisé ou dont le fondement est nébuleux. Malgré ses inévitables imperfections et chevauchements conceptuels, cette grille se révèle peut-être un peu plus fine que celles proposées par d'autres chercheurs (tableau 1$)^{6}$.

6. Evelyn Kolish a fait usage de quatre catégories générales afin d’analyser le contentieux civil de la fin du XVIII ${ }^{\mathrm{e}}$ siècle et du début du XIX ${ }^{\mathrm{e}}$ siècle : les personnes (incluant le droit familial); les immeubles; les héritages et donations; la responsabilité civile et les contrats. Sa quatrième catégorie renferme ellemême trois sous-thèmes: les dommages, les contrats (incluant les prêts et les hypothèques) et les pour- 
Tableau I

Enjeux des poursuites

Cour supérieure, district de Trois-Rivières

Dossiers des matières civiles en général, I880- I885- 1890

\begin{tabular}{|l|c|c|l|c|c|}
\hline \multirow{2}{*}{ Enjeux } & \multicolumn{2}{|c|}{ Dossiers } & \multirow{2}{*}{ Enjeux } & \multicolumn{2}{c|}{ Dossiers } \\
\cline { 2 - 3 } \cline { 6 - 7 } & $\mathbf{n}$ & $\%$ & & $\mathbf{n}$ & $\%$ \\
\hline 1- Échanges marchands & 23 & 3,3 & 6- Déconfitures financières & 47 & 6,8 \\
\hline 2- Services professionnels & 13 & 1,9 & 7- Affaires familiales & 47 & 6,8 \\
\hline 3- Immeubles & 69 & 10,0 & 8- Régulation étatique & 35 & 5,1 \\
\hline 4- Contrats & 49 & 7,1 & $\begin{array}{l}\text { 9- Dommages matériels, } \\
\text { physiques et moraux }\end{array}$ & 75 & 10,9 \\
\hline $\begin{array}{l}\text { 5- Billets promissoires } \\
\text { et équivalents }\end{array}$ & 199 & 28,9 & 10- Divers et enjeux inconnus & 132 & 19,2 \\
\hline
\end{tabular}

Source: BAnQ, CMCQ,TPII S3 SS2 SSSI, dossiers de I880, 1885 et 1890

a.Actes d'obligation, lettres de change et chèques

Les dossiers ne comportant qu'une demande de bref d'assignation rendent compte du nombre relativement important d'affaires dont l'enjeu précis demeure inconnu. Par contre, dans bien des cas, on sait que le demandeur a requis ce bref en raison d'une dette dont le montant est spécifié, sans que l'origine de cette dernière ne soit explicitée. Ainsi, il appert clairement que l'intervention du tribunal est sollicitée le plus souvent pour forcer, si possible, le remboursement de dettes de tous ordres. Ce n'est pas exactement une surprise. Les transactions conclues par les justiciables constituent une réalité plus mouvante que, par exemple, le droit des personnes, à plus forte raison dans les communautés du XIX ${ }^{e}$ siècle. À ce titre, la fonction principale de la Cour supérieure, durant les

suites pour dette (incluant les billets promissoires et les lettres de change). Evelyn Kolish, «Some Aspects of Civil Litigation in Lower Canada, 1785-1825: Towards the Use of Court Records for Canadian Social History", The Canadian Historical Review, 70, 3 (1989): 341-343. Il nous semble difficile de distinguer les héritages du droit familial, comme la condition des individus dépend très étroitement, au XIX ${ }^{\mathrm{e}}$ siècle, des droits et obligations liés au patrimoine familial, patrimoine en bonne partie immobilier au demeurant. Pour sa part, le classement des dossiers de la Prévôté de Québec réalisé par John A. Dickinson présente aussi quelques difficultés. L'échange de denrées agricoles est distingué du commerce et un groupe social, les artisans, surgit au beau milieu des divers enjeux des réclamations. John A. Dickinson, Justice et justiciables : la procédure civile à la Prévôté de Québec, 1667-1759 (Québec, Les Presses de l’Université Laval, 1982), 121-122. 
années 1880, ne diffère pas de celle des tribunaux civils de la fin du XVIII ${ }^{\mathrm{e}}$ siècle et du début du XIX ${ }^{\mathrm{e}}$ siècle ${ }^{7}$.

Une donnée plus précise saute aux yeux: le contentieux de la Cour supérieure est formé pour près du tiers de demandes de paiement de billets promissoires et d'effets semblables ${ }^{8}$. Sa tâche de régulation sociale concerne ainsi, au premier chef, les promesses formelles de paiement. Malheureusement, les bénéficiaires de billets promissoires n'ont pas à mentionner, lors d'une poursuite, la considération en vertu de laquelle ces effets ont été signés. La preuve documentaire suffit. Et alors que les marchands et commerçants forment la clientèle principale de la cour, côté poursuite, les litiges qui peuvent clairement être reliés à l'achat de marchandises (catégorie 1) s'avèrent plutôt rares. Il y a lieu de croire que plusieurs de ces ventes, pour des montants importants - eu égard au seuil monétaire de la juridiction du tribunal, soit $200 \$$ - ont été conclues contre une promesse de paiement "garantie" par la signature d'un billet. Certaines affaires en témoignent ${ }^{9}$.

Pour le reste, les poursuites en dommages $(10,9 \%)$ et les litiges immobiliers $(10,0 \%)$ forment une part non négligeable des causes entendues. Suivent, dans l'ordre, les causes contractuelles $(7,1 \%)$, les affaires familiales $(6,8 \%)$, les déconfitures financières $(6,8 \%)$ et les procédures relatives au fonctionnement des rouages de l'État (5,1\%). Le solde de comptes de marchandises (3,3\%) et les honoraires professionnels en souffrance $(1,9 \%)$ occupent plus rarement le personnel de la cour. Si ces données rendent compte grosso modo de la litigiosité civile d'envergure dans le district de Trois-Rivières à l'époque étudiée, les points de comparaison manquent, que ce soit en regard d'autres époques ou d'autres districts. Qui plus est, que signifient-elles en regard des pratiques courantes des justiciables? Il y a une marge importante entre ces pratiques et leur judiciarisation: 7\% de causes contractuelles, est-ce beaucoup? Et le marasme économique des années 1880 influence-t-il le nombre et la nature des réclamations? Ces questions demeurent en suspens. L'analyse qualitative des dossiers judiciaires offre toutefois des possibilités intéressantes. Cette approche permet de mieux connaître les enjeux sociojuridiques qui structurent le parcours des habitants du district. Il s'agit, de ce point de vue, d'identifier

7. E. Kolish, «Some Aspects of Civil Litigation in Lower Canada», loc. cit., 351.

8. Les trois quarts des litiges de la catégorie 5 concernent des billets promissoires.

9. Bibliothèque et Archives nationales du Québec (dorénavant BAnQ), Centre d'archives de la Mauricie et du Centre-du-Québec (dorénavant CMCQ), fonds des dossiers des matières civiles en général de la Cour supérieure, greffe de Trois-Rivières (fonds dorénavant désigné par sa cote, soit TP11 S3 SS2 SSS1), 1885, no 243, Valentine vs Plante. 
les liens de droit qui vont de pair avec les différents systèmes de production, de reproduction et de domination à l'œuvre dans cette société régionale. Faute d'espace, nous nous pencherons seulement sur cinq catégories de litiges: les immeubles, les contrats, les billets promissoires, les affaires familiales et les dommages.

\section{LES POURSUITES IMMOBILIÈRES}

Le corpus renferme 69 poursuites concernant directement des immeubles, soit exactement $10 \%$ du total de causes. Figurent ici des conflits relatifs à des transactions immobilières, au droit de propriété ou d'usage de biens fonciers ainsi qu'aux hypothèques affectant des terres, emplacements urbains et bâtiments. La circulation des immeubles sur le marché fut au cœur des changements apportés au droit civil durant le second tiers du $\mathrm{XIX}^{\mathrm{e}}$ siècle. Le contentieux immobilier de la Cour supérieure de la décennie 1880 permet de prendre la mesure des transformations qu'ont subies ou non, en pratique, les rapports sociaux et juridiques liés à la propriété immobilière.

Les dossiers de la Cour supérieure signalent une double complexité: complexité des pratiques au sujet des immeubles et complexité des liens sociojuridiques concomitants. S'il y a un marché de la terre en Mauricie durant la décennie 1880, c'est sous une forme particulière, marquée par la présence de réseaux compliqués d'ayants droit. En outre, les transactions portant sur des créances nées des divers modes de transfert de la propriété foncière montrent une distance importante envers l'idéal libéral d'un exercice absolu du droit de propriété.

La sûreté des titres demeure un enjeu important. On transige sur des terres et bâtiments toujours affectés de certaines charges. D’où, notamment, des débats à propos de la radiation d'hypothèques ${ }^{10}$. Surtout, le marché immobilier entre parfois en collision avec les pratiques patrimoniales des familles. Il n'y pas qu'un marché des immeubles. Des parts d'héritages sont vendues, parts parfois aussi petites que des $1 / 11^{\mathrm{e}}$ de moitié de terre. Surgissent alors des cas de propriété indivise. L'achat d'une ferme a certainement causé bien des maux de tête à François Cloutier, cultivateur de la paroisse de Sainte-Perpétue. Il est poursuivi par un hôtelier, Victor Dessert, au début du mois d'octobre 1885. Il faut remonter à 1859 , lors du mariage de Joseph et Marguerite Therrien en communauté de biens, pour comprendre l'affaire. Joseph a acquis une 
terre en 1872 ; sa femme est décédée l'année suivante. Or, leur contrat de mariage stipulait que le survivant aurait la jouissance des actifs de la communauté, à condition d'en faire inventaire. Ce qui n'a pas été fait.

Une communauté de biens aurait donc continué à exister entre le veuf et ses enfants. En 1875, Joseph Therrien vend la terre acquise en 1872 à Moïse Therrien. C'est probablement son fils. Cette transaction est-elle légale? Il possédait toujours l'immeuble en commun avec ses enfants et ne pouvait prétendre qu'à un usufruit - ou droit de jouissance - sur une moitié du lot. Moïse Therrien revend le tout en 1883 à Cloutier. Les choses se corsent en mars 1885 . Certains enfants du couple Therrien transportent leurs droits dans la succession de leur mère à Victor Dessert. Ces droits successoraux portent sur la moitié de l'immeuble. Résultat: Dessert se dit propriétaire des $3 / 10^{\mathrm{e}}$ de la moitié de l'immeuble, alors que Cloutier serait propriétaire des $7 / 10^{\mathrm{e}}$ de l'immeuble pris dans son ensemble. C'est la foire d'empoigne. En août, probablement alerté, Cloutier cesse ses paiements à Moïse Therrien qui le poursuit de ce fait. Dessert, de son côté, poursuit Cloutier en octobre pour concrétiser ses achats de droits successoraux: l'immeuble doit être partagé ou vendu. Cloutier, pour sa part, se retourne peu après contre Therrien en tant que demandeur en garantie, afin que ce dernier réponde de ce que lui, Cloutier, pourrait devoir à Dessert. Le $1^{\text {er }}$ juin 1886, le juge Bourgeois donne raison à la fois à Dessert (pour ses $3 / 10^{\mathrm{e}}$ ) et à Cloutier, qui se retrouve créancier de Moïse Therrien ${ }^{11}$.

Les archives de la Cour supérieure révèlent l'existence d'un véritable marché des créances hypothécaires. James Brulé a acheté un moulin en 1872 à Olivier Dostaler fils. Ce dernier l'avait lui-même acquis de son père qui lui-même devait encore de l'argent au propriétaire antérieur, Burn. D'où l'existence d'une hypothèque en faveur de la veuve de Burn et de ses enfants. Olivier Dostaler fils a ensuite cédé à la famille Burn ce que Brulé lui devait, Brulé se retrouvant débiteur des Burn. Les Burn ont subséquemment transporté leur créance à Ezekiel Hart, courtier et agent collecteur. Il réclame les sommes en souffrance à Brulé ${ }^{12}$.

Il y a une absence significative parmi les affaires immobilières, celle des banques. L'institutionnalisation du marché immobilier relève essentiellement de la pratique des notaires et des règles d'enregistrement, ce qui

11. BAnQ, CMCQ, TP11 S3 SS2 SSS1, 1885, $\mathrm{n}^{\circ} 186$, Therrien vs Cloutier; 1885, $\mathrm{n}^{\circ} 202$, Dessert vs Cloutier; $1885, \mathrm{n}^{\circ} 238$, Cloutier vs Therrien.

12. BAnQ, CMCQ, TP11 S3 SS2 SSS1, 1880, n 156, Hart vs Brulé. 
accentue le rôle de l'appareil judiciaire sur le plan de la régulation des transactions de ce type.

\section{LA « LIBERTÉ » DE S'ENGAGER : LES CONTRATS ET BILLETS PROMISSOIRES}

Notre échantillon ne contient qu'un petit nombre de causes contractuelles, soit 49 (7,1\% du total). L'inobservation ou l'interprétation d'une prestation ayant un objet clairement établi et consignée dans une entente formalisée est à l'origine du litige. La refonte du droit civil au milieu du $\mathrm{XIX}^{\mathrm{e}}$ siècle a été marquée par l'idéologie contractuelle, le contrat étant considéré comme l'expression la plus pure de la liberté d'entreprendre et de la rationalité économique des citoyens mâles, expression chère au libéralisme économique et aux élites qui la voulaient sans entraves. C'est aussi, plus concrètement, un des rouages essentiels du développement du marché. Les codificateurs donnèrent plus d'ampleur à la liberté contractuelle et à la sûreté des obligations concomitantes ${ }^{13}$. Bien entendu, les préoccupations des élites et les modifications apportées au droit civil ne signifient pas que les habitants de la province se sont mis subitement à "l'heure contractuelle». Ce n'est pas encore le cas dans les années 1880 en Mauricie, à moins que le formalisme allant de pair avec la signature de contrats ait fait obstacle, le plus souvent, à la judiciarisation des rapports contractuels.

Les quelques dizaines de litiges de cet ordre sont tout de même significatifs. L'économie du district judiciaire, durant la décennie 1880, met en relation des producteurs locaux et des intermédiaires impliqués dans des réseaux commerciaux régionaux ou suprarégionaux, et même des capitalistes d'envergure. Plusieurs conventions portant sur la livraison de quantités importantes de foin ou de bois conduisent leurs signataires devant le tribunal. Le commerce du bois n'est pas seulement l'apanage des magnats de la forêt mauricienne comme les Baptist et les Hall. Certains cultivateurs se font pour un temps petits entrepreneurs au profit d'hommes d'affaires plus importants qu'eux. Disposant de peu de capitaux, ils dépendent des avances fournies par leurs partenaires afin de lancer les travaux. Les voilà d'emblée débiteurs, situation assez risquée. L'inégalité des contractants est manifeste. Edward Calvin Goodhue, commerçant du village de Danville, poursuit Télesphore et Johnny Melançon de Saint-Léonard. Leur contrat, paraphé en août 1884, prévoyait la livraison de billes de pruches. Si Johnny était 
présent lors de la signature du contrat, Télesphore aurait oublié de le signer, tout en commençant à l'exécuter. D'après Goodhue, ses avances en argent et en marchandises ont une valeur supérieure au bois finalement livré, pour un montant de 157 \$. Les Melançon sont condamnés après avoir fait défaut de comparaitre ${ }^{14}$.

Les dossiers de la Cour supérieure recèlent bien quelques conflits au sujet du droit d'exploitation d'appareils et de brevets, signe de la mécanisation des activités de production, mais ces affaires ne concernent pas de grandes entreprises. Ils ne contiennent aussi que des traces très éparses des deux formes modernes de rapport à l'argent que sont les parts d'entreprise et les assurances. Au surplus, seules 6 causes contractuelles parmi les 49 affaires de cet ordre impliquent des entreprises incorporées. Les engagements contractuels, tout comme les transactions immobilières, sont encore faiblement institutionnalisés. Le fonctionnement du marché et du capitalisme dépend encore de rapports interindividuels.

La Cour est plus souvent appelée à entendre des réclamations relatives à d'autres types d'engagements formels que les contrats : son contentieux est constitué à près de $30 \%$ de poursuites concernant des billets promissoires ou des effets semblables (199 dossiers, 28,9\% du corpus). Quarantecinq réclamations fondées sur des actes d'obligation ont été incluses dans cette catégorie. Il s'agit clairement, dans le cas des actes d'obligation, de prêts à intérêt ou du moins des intérêts sont-ils à peu près toujours mentionnés ${ }^{15}$.

Les procès de ce genre ont leurs particularités. Ils se terminent le plus souvent par un jugement en bonne et due forme, cela plus fréquemment que d'autres affaires. Ils s'avèrent aussi assez expéditifs. Près de la moitié des décisions sont rendues par défaut, sans que le défendeur ne comparaisse. Comme les demandeurs n'ont pas à indiquer les circonstances entourant la signature des billets dont ils sont les bénéficiaires, la considération en vertu de laquelle le billet a été offert demeure un mystère dans bien des cas. Il faut par conséquent s'en remettre à des données plus périphériques pour aborder cette catégorie de procès: la mention ou l'absence de mention d'intérêts; la circulation plus ou moins étendue des billets; le rôle joué par les institutions bancaires. La prise en compte de ces facteurs permet de reconstituer une partie des rapports sociojuridiques

14. BAnQ, CMCQ, TP11 S3 SS2 SSS1, 1885, $\mathrm{n}^{\circ}$ 166, Goodhue vs Melançon.

15. BAnQ, CMCQ, TP11 S3 SS2 SSS1, 1890, $\mathrm{n}^{\circ}$ 200, Hart vs Bellefeuille. Nous avons également compté ici les rares réclamations concernant des chèques et des lettres de change. 
tissés autour de l'argent - ou des promesses de payer, pour être plus exact - dans le district de Trois-Rivières à la fin du XIX siècle.

Dans près de 75 dossiers, la requête n'évoque pas d'intérêts comptabilisés avant l'échéance du billet. Plusieurs possibilités se présentent: il s'agit d'un prêt sans intérêt; le capital réellement prêté est inférieur à la valeur du billet et les intérêts ont été inclus dans la somme à remettre à échéance ; le billet a servi à autre chose qu'un prêt, comme dans le cas du paiement d'une transaction. Peu importe, des intérêts commencent à courir après l'échéance. Pierre Calixte Neault, cultivateur, poursuit un autre cultivateur de sa paroisse. Le 13 septembre 1890, Joseph Dostaler a signé à son ordre un billet promissoire de $100 \$$ payable à un mois. Est-ce un prêt d'urgence que Dostaler croyait pouvoir rembourser à la suite d'une prochaine rentrée d'argent? Comme il n'a pas été payé, Neault réclame ses $100 \$$, additionnés d'intérêts de $6 \%$ comptabilisés à partir du 16 octobre $1890^{16}$.

Certains dossiers comportent un détail intéressant: le billet est payable à demande. Lorsqu'une échéance précise est stipulée, le porteur du titre ne peut faire appel à la justice qu'à partir de cette date. Un détenteur de capitaux désireux de resserrer son emprise sur son obligé pourrait préférer le paiement à demande. Arthur Turcotte Genest, éditeur et imprimeur, a signé deux billets à l'ordre de Gertrude Mary Devlin, dame célibataire de Trois-Rivières. Le premier, du 28 juin 1890, vaut 200 \$, somme payable à deux mois d'échéance; le second, signé seulement deux semaines plus tard, soit le 12 juillet 1890 , vaut $112 \$$ et son paiement peut être requis n'importe quand. Genest a-t-il réitéré trop rapidement ses demandes d'argent auprès de Mme Devlin, rendant celle-ci plus circonspecte? De fait, la poursuite qu'elle intente le $1^{\text {er }}$ septembre 1890 , pile à l'échéance du premier billet, précise que Genest a déjà payé $65 \$$ afin de solder sa deuxième obligation, la plus contraignante ${ }^{17}$.

Les transactions comportant des intérêts comptabilisés à partir de la date de signature du billet concerneraient le plus souvent des prêts et non des opérations commerciales ou entrepreneuriales. L'inégalité de la condition sociale du faiseur et du bénéficiaire du titre constituerait un bon indice d'activités de prêt à intérêt, tout comme la proximité géographique des parties. Les rapports unissant le marchand Pierre Nolasque Chaillez à des cultivateurs de sa paroisse sont évocateurs. Certains des billets en 
sa possession sont payables à demande et engendrent des intérêts de $8 \%$ par an; aucun de ces titres n'a circulé en d'autres mains que les siennes. C'est là se donner la latitude nécessaire à une bonne et prudente fructification des capitaux... On peut laisser les gains attendus courir plusieurs années. Chaillez ne s'en prive pas, tout en se réservant la possibilité de rappeler les créances au moment voulu ${ }^{18}$. Il est un aspect du rapport à l'argent, dans ce cas-ci, sur lequel les archives judiciaires demeurent malheureusement muettes. Le fait d'être marchand et d'habiter la même paroisse que ses obligés permet à Chaillez, sans doute, de surveiller la santé financière des emprunteurs, individus dont il a certainement une connaissance personnelle. Il dispose probablement des informations nécessaires pour déterminer si le moment de poursuivre est venu, le risque étant d'agir trop tard à l'encontre de concitoyens complètement insolvables.

La fréquence des transports de billets témoigne de leur rôle important dans la socio-économie du district. S'il reste fort à faire pour recréer le tissu d'obligations, de créances et d'engagements de toutes sortes reliant les uns aux autres les individus dans les communautés du passé, les dossiers de billets promissoires impayés sont riches d'enseignements à cet égard. Un billet cédé par le preneur à des tiers entre en circulation sur le marché local. Ce que les justiciables s'échangent, il faut le rappeler, ce sont des promesses de payer, obligations liant des personnes qui, le plus souvent, se connaissent et se côtoient d'assez près peut-on croire. Des tractations, dont les dossiers judiciaires ne portent pas la trace, ont peutêtre eu lieu afin de régler au moyen d'une seule procédure les multiples manquements d'un débiteur. Si le marchand Pierre Héroux poursuit en avril 1885 le cultivateur Euchariste Garceau pour quatre billets que ce dernier a signés à son ordre la même journée, il inclut dans sa requête le billet signé par Garceau à l'ordre de Frédéric Pellerin et que ce dernier lui a transporté ${ }^{19}$.

Nous n'avons recensé aucune poursuite intentée par une banque désireuse de recouvrer un prêt qu'elle aurait accordé. Le marché du prêt, dans le district de Trois-Rivières des années 1880, est tributaire de relations sociales de proximité. Les banques sont néanmoins présentes dans le contentieux de billets promissoires. Elles entrent en scène, habituellement, à la fin de la trajectoire des billets, lorsque des porteurs les leur

18. BAnQ, CMCQ, TP11 S3 SS2 SSS1, 1890, $\mathrm{n}^{\circ} 247$, Chaillez vs Thiffault; 1890, $\mathrm{n}^{\circ} 248$, Chaillez vs Brouillet.

19. BAnQ, CMCQ, TP11 S3 SS2 SSS1, 1885, n 95, Héroux vs Garceau. 
cèdent afin de se faire payer la somme. Ce qui est une forme d'octroi de crédit, bien entendu. Cette présence périphérique des banques témoigne du faible degré d’institutionnalisation du marché des créances, marché normé essentiellement par les règles du Code en matière d'obligations et par les règles de procédure civile, en cas de recours en justice. Si le billet n'est pas payé à échéance, l'institution émet un protêt. Deux possibilités s'offrent alors. Soit le porteur du titre paie l'institution et se retourne contre le faiseur et, s'il y en a, contre les anciens bénéficiaires qui se sont échangés le billet. Soit le dernier porteur ne paie pas et la banque le poursuit en compagnie des autres individus impliqués dans la création et la circulation du billet.

Les billets, souvent, naissent et circulent au sein des mêmes réseaux. Achille Gagnon, commerçant de bois, a maille à partir avec plusieurs banques durant l'année 1885 . Il a signé en sept jours quatre billets promissoires à l'ordre d'un autre Gagnon, Antoine, un arpenteur habitant comme lui à Arthabaskaville. Ces billets étaient payables à brève échéance au bureau de la Banque Union du Bas-Canada. Chaque fois, Antoine a transporté les billets à l'institution. Près de 45 jours après la signature des premiers billets, Antoine a lui-même signé un billet à l'ordre d'Achille, billet payable au bureau de la même banque et cédé à celle-ci. Il n'y eut jamais de fonds pour solder tous ces titres formant la somme colossale de près de $16000 \$ \$^{20}$. Achille a mené le même genre d'opérations avec le protonotaire du district d'Arthabaska, Bazile Théroux fils. Ce qui leur vaut une condamnation conjointe, cette fois aux mains de la Banque d'Hochelaga $^{21}$.

\section{LA RÉGULATION DE LA SPHÈRE PRIVÉE}

Deux types de litiges ont été comptabilisés parmi les affaires de famille, soit les poursuites intrafamiliales au sujet du patrimoine et les conflits qui, proprement relationnels, peuvent avoir en retour des conséquences considérables sur le rapport aux biens. Un jugement en séparation de corps entraînera par exemple le partage de la communauté de biens des époux mariés sous ce régime. Bien que peu nombreux (47), les litiges familiaux montrent que les différentes phases du cycle familial des populations d'autrefois présentent certains risques, risques personnels, financiers et juridiques tout à la fois. Des individus placés sous tutelle durant leur

20. BAnQ, CMCQ, TP11 S3 SS2 SSS1, 1885, $\mathrm{n}^{\circ}$ 107, Banque Union du Bas-Canada vs Gagnon.

21. BAnQ, CMCQ, TP11 S3 SS2 SSS1, 1885, nº 109, Banque d’Hochelaga vs Gagnon. 
enfance contestent, une fois adultes, les comptes soumis par leur tuteur; des femmes mariées se trouvent contraintes de réclamer une séparation de corps ou de biens; les dispositions prises pour transmettre un patrimoine dégénèrent à l'occasion en poursuites.

Une contestation de compte de tutelle peut se trouver mêlée aux autres stratégies de constitution et de dévolution du patrimoine familial. François Boisvert, cultivateur, s'est marié en séparation de biens en 1867 avec Marguerite Lajoie. Leur contrat comportait un douaire de 1000 en faveur de cette dernière. Dans son testament rédigé en 1881, François lègue ses avoirs à deux de ses fils. Il meurt très peu de temps après et sa veuve se remarie l'année suivante avec Dionis Lacerte, autre cultivateur. En juillet 1890, Marguerite poursuit l'un des deux héritiers, Adélard Boisvert. Ce dernier, cultivateur également, est majeur depuis moins d'un an. Pas de chance pour lui, François l'a chargé dans ses dernières volontés de payer le douaire de sa mère et a sécurisé ce paiement au moyen d'une hypothèque sur une moitié de terre qu’il lui a léguée. Or, le couple LajoieLacerte réclame le paiement du capital du douaire et des intérêts accrus depuis le décès du de cujus, pour un total de 1540 \$. Adélard rétorque que les demandeurs n'ont droit qu'à l'usufruit de la somme. Surtout, il remet en cause l'honnêteté du compte de tutelle que lui ont soumis sa mère et son second mari. Ces derniers ont administré ses biens durant sa minorité. Simple coïncidence? Ce compte a été produit en juin 1890, peu de temps avant l'institution de leur action. Ils auraient exagéré de beaucoup les dépenses et omis des recettes importantes. Le duo, à ses yeux, lui devrait près de $3500 \$$.

La réclamation en paiement du douaire est accueillie par le juge le 21 mai 1891, après confession du défendeur pour $1000 \$ \$ 22$. Adélard Boisvert ne s'est pas contenté de répondre à cette action. Il a fait du reliquat du compte de tutelle l'objet d'une poursuite en bonne et due forme. La défense offerte par le couple durant cet autre procès ne manque pas de couleur: François Boisvert, père du demandeur, aurait été un mauvais cultivateur, dépensier de surcroît. Les dettes laissées en plan à sa mort expliqueraient le passif du compte de tutelle. Si le juge tranche en faveur d'Adélard le 16 avril 1891, c'est seulement pour le dixième de la somme réclamée, soit $370,51 \${ }^{23}$. Il se retrouve donc, au final, débiteur de sa mère et de son beau-père pour près de $630 \$$. Il s'agit là d'un bel exemple de la 
manière dont le patrimoine et les décisions prises à son sujet (comme refiler à un fils un engagement pris en contrat de mariage !) pèsent parfois lourdement sur les rapports familiaux.

Le règlement d'une succession peut aussi compliquer la conduite des affaires. Il n'y a pas qu'en milieu rural que le patrimoine structure les rapports familiaux. Les activités capitalistes ont souvent une importante composante familiale, ce qui ajoute au caractère très interpersonnel du rapport à l'argent. Margaret Baptist est la fille de George Baptist, magnat de la forêt mauricienne décédé en 1875 . En 1880, elle réclame $2216,92 \$$ à James Dean et à John Baptist. Cette somme représente le total des intérêts échus sur sa part dans la succession de son père. Elle fait valoir que cette succession inclut la dette que les défendeurs ont contractée à l'endroit de George Baptist en s'associant à lui pour faire le commerce du bois, dette de près de 150000 \$. La George Baptist and Sons est une entreprise familiale. John est le fils de George, Dean son gendre. Ils répliquent que la mort de George n'a pas mis fin au contrat qui les liait de son vivant et que Margaret, en acceptant la succession de son père, a accepté les dettes de l'entreprise. En outre, la firme se trouverait dans l'impossibilité de rembourser ses créanciers en raison de la conjoncture économique défavorable. Le juge Gill renvoie leur défense. Ce qu'ils doivent à l'entreprise fait réellement partie du patrimoine du défunt et Margaret a droit aux intérêts sur son legs. Porté en appel, le jugement est confirmé par la Cour du banc de la Reine ${ }^{24}$.

\section{LES DOMMAGES MATÉRIELS, CORPORELS ET MORAUX}

Les poursuites en dommages de tous ordres sont bien représentées, avec 75 cas recensés (10,9\% des dossiers). Les défendeurs se voient reprocher leur négligence ou leur malice. Les sommes réclamées et éventuellement allouées par le juge, en ce domaine, ne sont pas aussi bien déterminées que lorsqu'une preuve documentaire établit clairement l'existence d'une créance. Les demandeurs et les avocats qui les représentent ne se gênent pas pour présenter des réclamations considérables, élans qu'un jugement favorable, le cas échéant, se chargera de tempérer, et de beaucoup.

Les empiètements sur des terres peuvent constituer une source de tensions assez vives parmi des populations qui, en grande partie, tirent encore leur subsistance du sol. Ces situations suscitent des débats sur le bornage de terrains contigus, notamment après l'arrachage de poteaux 
plantés (et replantés) par certains individus pour délimiter ce qu'ils considèrent être leur propriétée ${ }^{25}$. Fait surprenant de prime abord, on ne compte qu'une réclamation pour des dommages matériels résultant d'un accident $^{26}$. Plus étonnante encore est la quasi-absence de réclamations pour des dommages corporels. Comment se fait-il que la Cour supérieure n'entende aucune cause d'accident de travail durant les trois années échantillonnées? Or, la dangerosité des milieux de travail, à cette époque, n'est plus à démontrer. Et de tels accidents (mort d'un mari pourvoyeur, amputation d'un membre, etc.) sont certainement en mesure de générer des poursuites de plus de $200 \$$, seuil monétaire de la juridiction de la cour.

Il y a certes quelques réclamations pour des blessures subies lors de bagarres, affaires où se mêlent coups et insultes. La sensibilité des requérants a également été affectée et il est parfois difficile de départager les dommages corporels des blessures à l'amour-propre. Une séance de la Cour des commissaires de décembre 1885 s'est plutôt mal terminée. Deux individus en sont venus aux coups. Le demandeur soutient qu'il a été blessé et que l'esclandre a entaché sa réputation. Il réclame $250 \$$ et en obtient 50. La source du conflit? Une dette de $1,40 \$ \$^{27}$.

En matière d'atteinte à la réputation au sens strict, sans mention de brutalités concomitantes, les demandeurs se plaignent la plupart du temps de deux types de propos diffamatoires, soit d'allégations de fraude et d'insolvabilité, d'une part, et d'allégations d'inconduite sexuelle, d'autre part. La probité et l'habileté en affaires ainsi que la sexualité confinée au mariage constituent des assises importantes de la considération dont veulent jouir les justiciables du district durant la seconde moitié du XIX ${ }^{\mathrm{e}}$ siècle. Du moins sont-ce là des formes de renommée dignes d'être défendues par un appel à l'autorité de la Cour supérieure.

Les demandeurs et défendeurs des procès en diffamation appartiennent souvent aux mêmes classes sociales et résident la plupart du temps à proximité les uns des autres. Cela traduit la sociabilité propre à la vie dans le district à l'époque. Les individus évoluent dans des communautés d'appartenance où la plupart des gens se connaissent (paroisses, villages et quartiers). Ces communautés d'appartenance constituent aussi, simultanément, les scènes où se jouent les réputations. Être mal considéré par les habitants de sa paroisse s'avère un réel problème, car c'est en bonne partie à cette échelle que les obligations sont contractées, que s'élaborent

25. BAnQ, CMCQ, TP11 S3 SS2 SSS1, 1885, $\mathrm{n}^{\circ}$ 182, Pérusse vs Marchand.

26. BAnQ, CMCQ, TP11 S3 SS2 SSS1, 1885, $\mathrm{n}^{\circ} 71$, Courtois vs Cloutier.

27. BAnQ, CMCQ, TP11 S3 SS2 SSS1, 1885, n² 264, Caron vs Gerbeau. 
les stratégies matrimoniales des familles et, plus généralement, que l'on est vu et reconnu.

Dans une économie encore largement fondée sur des rapports de face-àface et sur le crédit, le crédit, au sens de confiance inspirée, est un facteur clé de la conduite des affaires et plus généralement de l'intégration sociale des justiciables. Un forgeron, Ludger Houle, a maille à partir avec un cultivateur, Emmanuel Provencher. Ce dernier, dit-il, l'a présenté comme un faussaire et a fait courir le bruit qu'il se préparait à s'enfuir aux États-Unis pour échapper à la justice. Provencher est son oncle, il faut le préciser. Ces allégations, dit Houle, ont nui à son crédit et à sa réputation, car elles sont devenues de notoriété publique dans les paroisses où habitent les deux hommes. Il réclame $1000 \$$. Tout en niant avoir tenu ces propos, Provencher soutient que si cela a été le cas, c'est qu'il les a entendus de tierces personnes. Il n'en est donc pas à la source. L'action est renvoyée pour insuffisance de preuves $^{28}$.

Dans une société fondée sur la famille et où les normes morales et religieuses imprègnent le quotidien des acteurs sociaux, les rumeurs d'inconduite sexuelle ont une résonance particulière. Le fait de traiter de prostituée la femme d'un concitoyen est une injure on ne peut plus grave. Un cultivateur poursuivi sur ce chef, tout en se défendant d'avoir fait circuler de tels propos, fait valoir que la réputation de la dame concernée était déjà mauvaise, ce qui n'empêche pas le juge de le condamner à $20 \$$ de dommages sur les $500 \$$ réclamés par un autre cultivateur de la même paroisse $^{29}$.

\section{CULTIVATEURS ET INDUSTRIELS EN PROCÈS}

Inversons maintenant l'approche. Au lieu d'examiner la signification de certains types de procès, nous tiendrons compte de la position dans le contentieux du tribunal de deux groupes socioprofessionnels, les cultivateurs et les industriels. Ces deux groupes ont été choisis à dessein. La terre et l'agriculture étaient le socle des sociétés préindustrielles alors que la production manufacturée se trouve à l'avant-garde de la transformation de la socio-économie du district durant les années 1880.

Les 1830 parties du corpus renferment un gros contingent de cultivateurs: ils y apparaissent à 402 reprises. Ils sont nettement plus souvent défendeurs (253 rôles de défendeur, soit $62,9 \%$ des rôles joués par les 
cultivateurs) que demandeurs (149 rôles de demandeur, soit 37,1\% des rôles joués par les cultivateurs). On les retrouve le plus souvent dans des affaires de billets promissoires impayés, et ce, massivement du côté de la défense. De condition modeste pour la plupart, les cultivateurs doivent probablement plus souvent qu'à leur tour requérir des prêts et avoir du mal à les rembourser, même si les billets promissoires ont d'autres usages. Toujours est-il qu'ils ne sont pas absents du marché de l'argent et des créances, puisque d'autres justiciables ont manifestement accepté des billets signés de leur main.

Le portrait est le même, de manière générale. Des cultivateurs sont poursuivis après avoir signé de tels billets à intérêt ou actes d'obligation à intérêt au profit de personnes de condition sociale supérieure à la leur. Répétons-le, les dossiers judiciaires ne dévoilent que rarement la considération en vertu de laquelle un billet est signé. Dans bien des cas, le temps écoulé entre la signature du document et le recours en justice a pour effet que le montant réclamé en Cour supérieure dépasse, et de loin, la somme dont le billet prouve la promesse de paiement. Des transactions versent carrément dans l'usure, tels ces actes d'obligation portant $16 \%$ d'intérêt annuel et chargés d'une clause stipulant qu'en cas de défaut de paiement annuel des intérêts, ceux-ci seraient capitalisés... et engendreraient à leur tour des intérêts de $16 \%$. La veuve d'un «bourgeois » poursuit sur ce chef un couple de cultivateurs et leur fils, navigateur de métier ${ }^{30}$. D’autres dossiers font état de transactions multiples entre cultivateurs et commerçants et dévoilent du même coup la dépendance des premiers envers les seconds. Euchariste Garceau doit solder cinq billets promissoires auprès d'un marchand ${ }^{31}$.

Cette situation globalement défavorable des cultivateurs est démentie en de rares occasions. Certains sont prospères et jouissent d'une situation plus enviable que des boutiquiers ou cols blancs. Les gens des campagnes ne constituent pas un groupe monolithique de producteurs primaires exploités; le monde rural a ses propres hiérarchies. L'épouse séparée de biens d'un cultivateur poursuit deux autres cultivateurs pour près de 1400 \$, somme substantielle, en raison d'un prêt consenti par le biais d'un acte d'obligation échu depuis deux ans et demi ${ }^{32}$.

Des cultivateurs sont aussi affectés par l'échec de donations. Certaines ont déraillé puisque les donataires ont quitté la région, comme bien d'autres

30. BAnQ, CMCQ, TP11 S3 SS2 SSS1, 1880, $\mathrm{n}^{\circ}$ 39, Gendle vs Lemire.

31. BAnQ, CMCQ, TP11 S3 SS2 SSS1, 1885, $\mathrm{n}^{\circ} 95$, Héroux vs Garceau.

32. BAnQ, CMCQ, TP11 S3 SS2 SSS1, 1890, $\mathrm{n}^{\circ}$ 304, Rouleau vs Dumas. 
ruraux à l'époque, pour tenter leur chance aux États-Unis. François Bossu dit Lyonnais avait chargé son fils Thomas de payer une rente à lui-même et à sa femme et de prendre soin de ses frères. Thomas ayant émigré aux ÉtatsUnis, Bossu dit Lyonnais souhaite que la donation soit révoquée et que ses biens meubles et immeubles lui soient rétrocédés ${ }^{33}$.

Des 1830 parties recensées, demanderesses comme défenderesses, 162 appartiennent au groupe des industriels et manufacturiers ( $8,9 \%$ du total). Leur représentation dans le contentieux de la Cour supérieure dépasse certainement, toutes proportions gardées, leur poids au sein de la population active du district. Les grosses affaires que brassent plusieurs d'entre eux en font des usagers tout désignés de la Cour supérieure. Leur positionnement judiciaire est à l'inverse de celui des cultivateurs: ils sont nettement plus souvent poursuivants (96 occurrences, soit 59,3\% des rôles joués par les industriels et manufacturiers) que poursuivis (66 occurrences ou $40,7 \%$ ). On ne peut pas voir en eux, cependant, un groupe social cohésif qui exercerait sa domination par le biais du tribunal. Des conflits opposent des entrepreneurs les uns aux autres et certains se retrouvent en mauvaise posture en raison des aléas de la conjoncture.

Le contentieux des industriels et manufacturiers renvoie à un aspect crucial du fonctionnement du marché : ses différents jeux d'échelle. On a droit à quelques surprises. Comment les frères Gault, les maîtres pancanadiens de la production textile, en sont-ils arrivés à prendre possession de deux billets promissoires de $139 \$$ dont le faiseur est une marchande publique de Trois-Rivières ${ }^{34}$ ? Les rapports commerciaux avec des capitalistes de Montréal suscitent des poursuites, comme celles mettant aux prises les Dawes, magnats montréalais de la bière, et les hommes d'affaires beaucoup plus modestes que sont un hôtelier et un marchand de vin du district ${ }^{35}$.

Le capitalisme fait surgir bon nombre de corps intermédiaires, sociétés ou entreprises de toutes tailles. De ce fait, les pratiques contractuelles peuvent conduire à des imbroglios. Étienne Beauchemin, cultivateur de la paroisse de Sainte-Monique, a vendu le droit de prélever l'écorce de pruche sur deux lots à des entrepreneurs du Massachusetts. La vente a été garantie par une hypothèque sur les deux mêmes immeubles. Il soutient avoir rempli ses obligations et requiert en 1885 la radiation de l'hypo-

33. BAnQ, CMCQ, TP11 S3 SS2 SSS1, 1880, $\mathrm{n}^{\circ}$ 13, Bossu dit Lyonnais vs Bossu dit Lyonnais.

34. BAnQ, CMCQ, TP11 S3 SS2 SSS1, 1885, $\mathrm{n}^{\circ}$ 90, Gault vs Sharples; 1885, $\mathrm{n}^{\circ}$ 206, Gault vs Sharples.

35. BAnQ, CMCQ, TP11 S3 SS2 SSS1, 1885, $\mathrm{n}^{\circ} 211$, Dawes vs Desaulniers; 1890, $\mathrm{n}^{\circ}$ 528, Dawes vs Dupont. 
thèque qui, toujours pendante, lui causerait par là des dommages. La société impliquée dans le contrat original, E. Bradley and Co., aurait confié par contrat la représentation de ses intérêts à une autre entité, la Young, Walton and Co. Les défendeurs, John Young et Arthur Walton, poursuivis personnellement, répliquent que ce n'est pas Young, Walton and Co. qui doit répondre de la poursuite, mais bien Young, Thayer and Co. En plus, la Young, Walton and Co. n'existerait plus depuis peu. La poursuite est renvoyée $e^{36}$.

Les liens d'affaires demeurent très interpersonnels, néanmoins. Les litiges mettant en scène des industriels et manufacturiers comportent une absence étonnante, celle des entreprises incorporées. Bien qu'elles soient évoquées de temps à autre, elles n'agissent à peu près jamais comme demanderesses ou défenderesses. Si on met de côté les autres personnes morales que sont les municipalités et les banques, il n'y a pas plus d'une vingtaine d'entreprises incorporées parmi les 1830 demandeurs et défendeurs de notre échantillon.

C'est au moment où certaines associations se délitent que le caractère interpersonnel du capitalisme local se dévoile avec le plus d'évidence. Les rapports entre les entrepreneurs forestiers George Ball et Francis McCaffrey s'aigrissent à la fin des années 1880. Ils faisaient affaire ensemble depuis 1876 et se sont mutuellement prêté de l'argent. Ball décide de balancer leurs comptes. Il réclame 2380,42\$ à son ex-partenaire le 6 février 1890. McCaffrey rétorque que Ball lui doit notamment près de $11000 \$$ pour avoir utilisé ses estacades de la rivière Nicolet. Ces infrastructures ont leur propre histoire, assez compliquée. McCaffrey aurait cédé à Antoine Mayrand (propriétaire d'une scierie à Nicolet) une partie de la propriété des estacades, tout en se réservant leur usage. Mayrand aurait à son tour transporté une partie des droits sur les estacades à un autre joueur, O'Shaugnessey. Deux clans s'affrontent: O’Shaugnessey et McCaffrey poursuivent Ball le 21 mars 1890 au motif que ce dernier détiendrait illégalement les estacades.

Le litige se complique. Les estacades sont à lui, affirme Ball, car il les aurait acquises d'un autre commerçant de bois, Ross. Il avait endossé ce dernier, les installations servant de garantie. De plus, Ball soutient les avoir construites lui-même en partie. La veille de cette contre-poursuite, soit le 20 mars, un marchand de la paroisse de Sainte-Clothilde-de-Horton a sauté dans la mêlée. Camille Gélinas exige que McCaffrey fasse cession de ses biens. McCaffrey, manifestement aux abois, se défend néanmoins 
d'être insolvable. Si ses paiements tardent, c'est que Ball lui doit de l'argent en vertu d'un jugement rendu par la Cour supérieure de Montréal à propos de l'usage des estacades. Fait inhabituel parmi les dossiers de faillite, plutôt sobres sur le plan discursif, il avance que Gélinas n'est qu'un prête-nom dont Ball se sert pour le persécuter. Cette requête en cession de biens ne semble pas aboutir, à la différence d'une démarche semblable entamée à la fin de l'année $1890^{37}$.

En d'autres temps, la propriété des moyens de production s'embrouille lorsqu'elle est mêlée aux stratégies matrimoniales et successorales. Par son testament de 1865, Alexis Gélinas a cédé la nue propriété de plusieurs moulins à ses trois fils. Sa veuve s'est retrouvée avec l'usufruit de ces immeubles. Avec le droit, donc, d'en tirer les revenus. Or, comme elle s'est remariée en communauté de biens en 1866, l'usufruit serait tombé dans cette communauté. En 1884, Blais, le second mari, a conclu une entente avec les fils du premier lit. Ceux-ci devaient dorénavant lui fournir la moitié des revenus tirés annuellement des moulins. Blais soutient n'avoir rien reçu. Alexis Gélinas fils, le défendeur, affirme avoir remis certaines sommes directement à sa mère. La poursuite est débouté $e^{38}$.

\section{CONCLUSION}

Nous avons donc examiné en détail, au cours de ces deux articles, les litiges soumis à l'attention de la Cour supérieure dans le district de TroisRivières durant les années 1880, 1885 et 1890. La condition socioprofessionnelle des parties et la nature des causes entendues ont fait l'objet d'un traitement systématique. Les dossiers des matières civiles en général ont aussi été mis à contribution afin de mieux comprendre la dynamique réunissant droit civil, justice et société durant la transition au capitalisme industriel. Il s'agissait d'une zone d'ombre de la littérature scientifique, tant en histoire sociale qu'en histoire du droit. Nous avons cherché, entre autres choses, à reconstituer l'expérience de la litigiosité civile en tenant compte simultanément des inégalités socioprofessionnelles, des pratiques des justiciables, des liens de droit en jeu dans les disputes qui les ont opposés et des prétentions qu'ils firent valoir auprès du tribunal.

La juridiction de la Cour supérieure fait en sorte que les plaideurs qui y croisent le fer présentent un profil sociologique particulier. Les commerçants forment sa principale clientèle, ce qui est symptomatique de

37. BAnQ, CMCQ, TP11 S3 SS2 SSS1, 1890, n 179, Ball vs McCaffrey; 1890, $\mathrm{n}^{\circ} 219$, O’Shaugnessey vs Ball; 1890, $\mathrm{n}^{\circ} 324$, Gélinas vs McCaffrey; 1890, $\mathrm{n}^{\circ}$ 529, Létourneux vs McCaffrey.

38. BAnQ, CMCQ, TP11 S3 SS2 SSS1, 1885, n 55, Blais vs Gélinas. 
l'importance de ce tribunal sur le plan de la régulation du marché des biens et de l'argent. Certains justiciables font appel assez fréquemment à son autorité, notamment des marchands et manieurs d'argent qui ont pour débiteurs plusieurs de leurs concitoyens en difficulté ou moins bien nantis. Certes, on ne peut voir en la Cour supérieure un instrument de domination de classe, compte tenu de la diversité des pouvoirs qu'elle exerce. Bien des conflits économiques, au demeurant, opposent des justiciables de même condition. Ses archives rendent compte néanmoins de la situation financière globalement défavorable des cultivateurs et de leur endettement chronique.

Ce tribunal gère aussi, en quelque sorte, le bouillonnant marché des créances véhiculées par des billets promissoires. Les réclamations relatives à ces effets de commerce forment près du tiers de son contentieux. Mais bien que l'argent ou plus exactement les promesses de paiement soient au cœur de ses activités, la Cour supérieure n'est pas seulement un organe étatique d'arbitrage des transactions de toutes sortes. Les justiciables y recourent en cas de dispute familiale grave ou pour défendre leur honneur, pour ne nommer que deux exemples. Surtout, on ne peut considérer isolément intérêts financiers privés, statut social des individus et trajectoires de ceux-ci. Être un débiteur impécunieux, un associé en difficulté ou une épouse affectée par la faillite de son époux constitue un dérèglement des liens juridiques, sociaux et culturels qui font la trame de la vie en société dans le district de Trois-Rivières durant les années 1880. Rendre justice, même pour un billet promissoire impayé, c'est également signifier ce qui en est de l'intégration et du positionnement des personnes au sein de communautés, réseaux d'affaires et familles.

Les dossiers judiciaires consultés renvoient à une phase particulière de l'histoire de la juridicisation et judiciarisation en matières civiles au Québec. La faible institutionnalisation des rapports économiques et sociaux fait des normes juridiques et des procédures judiciaires des rouages absolument centraux de la vie en société durant la seconde moitié du XIX ${ }^{e}$ siècle, ce qui remet en question les discours sur le trop-plein de droit et de recours aux tribunaux dans les sociétés contemporaines. À la différence de l'époque préindustrielle, les activités des tribunaux supérieurs s'étendent maintenant aux campagnes de la province durant les années 1880. Le droit et la justice ne sont pas alors concurrencés par d'autres pratiques institutionnalisées de fructification des avoirs et de gestion des aléas de la vie en société, par ces organes bureaucratiques qui domineront et prendront en quelque sorte en charge, en amont, la vie 
économique et le sort des particuliers. Au $\mathrm{XX}^{\mathrm{e}}$ siècle, au binôme droitjustice viendront s'ajouter d'autres entités quasi absentes du corpus en tant que parties: les grandes corporations industrielles et les institutions bancaires. Les premières s'empareront de la production et appropriation de la valeur au moyen du salariat et de l'actionnariat, formes plus impersonnelles de rapports sociaux. Les banques, elles, désocialiseront le rapport au crédit. Enfin, les assurances tant privées que publiques viendront dénouer en partie les nœuds de la reproduction sociale des familles et desserrer l'imbrication étroite entre droit, personnes et patrimoine.

Cette centralité du droit et de la justice, durant la décennie 1880, est nourrie en retour par la façon dont naissent les obligations de toutes sortes, de même que par la proximité entre justiciables. Le degré élevé d'interconnaissance est un facteur clé des rapports entre droit et société dans le district de Trois-Rivières à cette époque. Comme l'analyse de l'éventail de litiges l'a bien montré, tant le crédit, les activités entrepreneuriales, la reproduction sociale des familles - processus dominé par le patrimoine et les décisions prises à son sujet - que la manière dont la réputation est vécue contribuent à faire des liens de droit et de la judiciarisation des conflits des phénomènes interpersonnels, des réalités de faceà-face. Droit et justice ont une concrétude, une immédiateté considérable à l'époque. Et si les cultivateurs et les industriels, du fait de leurs activités et de leur condition sociale, présentent une litigiosité différente, ces deux catégories sociales expérimentent un peu le même rapport personnel au droit et à la justice. Des successions comprenant des terres, des parts d'entreprises et des moulins peuvent par exemple se traduire dans tous les cas par des poursuites mettant aux prises des apparentés.

On ne relève à peu près aucune trace, dans le corpus examiné, des réformes apportées au droit civil durant le second tiers du XIX ${ }^{\mathrm{e}}$ siècle au profit de la liberté contractuelle et d'un droit de propriété plus étendu. Non pas que nous nous attendions nécessairement à trouver de telles traces, cela pour de multiples raisons. Notamment, modifier le droit positif n'implique pas, évidemment, que les justiciables vont transformer de ce pas leurs pratiques. Et le cas échéant, ces pratiques neuves ne se traduiront pas nécessairement par des poursuites. Les normes juridiques formelles constituent une réalité bien distincte des conflits que les justiciables souhaitent ou se trouvent capables de traduire par une réclamation en justice. Ces disputes sont issues de rapports économiques et sociaux réels, immédiats, alors que la réforme du droit civil, au XIX ${ }^{\mathrm{e}}$ siècle, relève pour l'essentiel de décisions prises par les élites au sujet de ce que devait 
être, à leurs yeux, un droit civil adapté à la quête de profits par les citoyens mâles et répondant aux défis du capitalisme commercial et industriel.

Si le droit nouveau doit être socialement investi pour donner sa pleine mesure, ce sont les transformations des rapports socio-économiques au sein même des communautés qui permettront, peut-on croire, de donner toute sa portée au droit libéral du Code civil du Bas-Canada de 1866. À ce titre, la comparaison entre le contentieux des années 1880 et celui des années 1920 s'annonce prometteuse, puisque c'est durant cette dernière période que la grande industrie finira par dominer le district, tant en ce qui a trait à l'exploitation de la force de travail des populations qu'à l'extraction et transformation de ses ressources naturelles.

Conséquemment, réformer le droit ne vient pas changer «d'en haut» la manière dont, au quotidien, les individus et organisations produisent de la valeur, ni les différents systèmes de production, reproduction et domination. Par contre, le tribunal assume bel et bien une fonction cruciale: il met en œuvre le droit formel, il arbitre les disputes sous l'égide de ce dernier. L'essentiel est donc peut-être ailleurs. Les changements apportés au droit positif au milieu du XIX ${ }^{\mathrm{e}}$ siècle eurent peut-être moins d'importance, sur le plan de l'expansion du libéralisme économique, que les réformes institutionnelles qui ont permis d'assurer un règlement plus rapide des conflits découlant des engagements et obligations des justiciables, favorisant ainsi la marchandisation de la vie sociale. De ce point de vue, la décentralisation judiciaire de 1857 mérite autant d'attention, sinon plus, que le processus de codification entamé au même moment.

$\mathrm{Si}$, de fait, la cour arbitre le quotidien des disputes surgies des interactions économiques et sociales, elle sanctionne l’impéritie et le défaut de respect des engagements, tant dans la sphère privée que publique. Elle réduit ce faisant la profusion stochastique des aléas de l'économie et de la vie en société à des décisions basées sur des règles universelles; elle effectue une perpétuelle remise en ordre de l'état des personnes, des biens et des intérêts. C'est bien cette forme de régulation sociale qui se veut simultanément rationnelle, universelle et efficace qui fait du binôme droitjustice un instrument parfait de la modernité libérale. Les chercheurs devraient ainsi laisser un peu de côté, sans bien sûr les négliger, l'exégèse des règles de droit, les généalogies jurisprudentielles et les études de cas, afin de plonger dans le quotidien des activités des tribunaux et la masse de documentation "ordinaire» que ceux-ci ont laissée derrière eux. C'est peut-être de cette manière que la signification changeante du droit civil pour la société québécoise se donne le mieux à lire. 